\title{
Oblique High Resolution Tomography: The Ideal Plane for Visualization of the Gonial Section of the Mandibular Canal and its Related Structures?
}

\author{
Heinz-Theo Lübbers, MD, DMD ${ }^{1 *}$; Astrid L. Kruse, MD, DMD ${ }^{1}$; Joachim A. \\ Obwegeser, MD, DMD ${ }^{1}$; Klaus W. Grätz, Prof., MD, DMD ${ }^{1}$; Gerold Eyrich, MD, \\ DMD $^{2}$ \\ ${ }^{1}$ Clinic for Oral and Cranio-Maxillofacial Surgery, University Hospital Zurich, \\ Zurich, Switzerland \\ ${ }^{2}$ Private Practice for Oral and Cranio-Maxillofacial Surgery, Lachen, Switzerland
}

\begin{abstract}
A new radiologic technique is introduced in this paper for reducing the risk of nerve damage as a result of surgical removal of the mandibular third molar (wisdom tooth). The gonial part of the mandibular canal is obliquely scanned with tomograms on a plane parallel to this part of the mandibular canal. This procedure can be performed with the patient either prone or supine. The scans obtained cover a much longer section of the canal than the axial or coronal plane. Therefore, the scan provides more precise information on the spatial relationship between the mandibular canal and the surrounding structures with fewer images and, therefore, a lower radiation dose. Through such oblique plane scanning, metal artifacts from dental restorations do not impair visualization of the mandibular canal. Clinical cases demonstrating the advantages of this new technique are presented.
\end{abstract}

Keywords: cone beam computer tomography, multi slice computer tomography, oral surgery, wisdom tooth

\section{INTRODUCTION}

Surgical removal of impacted mandibular third molars (wisdom teeth) is probably one of the most frequent procedures in oral and maxillofacial surgery. The reasons for extraction may include chronic pericoronitis, the presence of a tumor or cysts, and carious or periodontal lesions. Complications associated with the removal of the lower third molars include infection, bleeding, and damage to the inferior alveolar nerve. The risk for nerve damage is well known, with results ranging from mild hypesthesia to major anesthesia. Several factors have been indicated in the literature as causes of nerve

*Corresponding Author: Heinz-Theo Lübbers, Clinic for Oral and Cranio-Maxillofacial Surgery, University Hospital Zurich, Frauenklinikstr. 24, 8091 Zurich, Switzerland. Phone: +41(44) 255-5064. Fax: +41 (44) 2554179.E-mail: Heinz-Theo.Luebbers@usz.ch. Other authors: Astrid.Kruse@usz.ch; Joachim.Obwegeser@usz.ch; Klaus.Graetz@usz.ch; Gerold.Eyrich@bluewin.ch. 
damage, such as deep impaction, surgeon inexperience, and age of the patient [1-3]. Susarala and Dodson [4] stated that nerve damage following third molar surgery happens in $1-22 \%$ of surgeries. This risk can be reduced if the surgeon has a thorough knowledge of the individual anatomy.

Preoperative radiological findings that indicate a close relationship to the nerve are well known and are described in conventional panoramic radiographs [5] as well as in three-dimensional (3D) radiography [4, 6-8]. With the development of computer tomography (CT) in 1972 [9, 10], 3D imaging prior to wisdom teeth removal became more and more routine [11-13]. The modern technique of cone-beam computer tomography (CBCT) seems to be significantly superior to panoramic radiographs in both sensitivity and specificity, therefore leading to a higher level of intrasurgical safety [12-15]. The open question remained whether or not the extra radiation dose for 3D-imaging was justified by the information gained $[12,13]$.

This paper describes a technique for multi-slice CT (MSCT), which reduces the scan field and therefore the radiation dose, eliminates accidental encounters with metal artifacts, and provides a view of long sections of the mandibular canal on each slice. Clinical test results demonstrating the advantages of this new technique are presented.

\subsection{The Oblique Radiological Technique}

With oblique scanning of the gonial section of the mandibular canal, the tomograms are made parallel to the arch-like contour of the latter (Figures 1 and 2a). Scanning is done with the patient either supine or prone, whereas a prone position with neck extended is most common. The gantry is angulated until the oblique plane is obtained. This oblique plane is neither a conventional coronal nor an axial plane; it is defined as the longest section of the curved path of the mandibular canal in the area of interest. It varies from patient to patient, and has to be identified in the scanogram (Figure 1). In case of different oblique planes for each side of the mandible, either a mean position is chosen or, if applicable, the side of interest determines the plane to choose.

With this technique, only a small number of slices are necessary, with a relatively small volume mostly between 1 and $2 \mathrm{~cm}$ in height. The oblique plane provides long cross sections of the mandibular canal on each slide (Figure 2a), in comparison to the standard axial (Figure 2b) or coronal (Figure 2c) planes. Furthermore, the oblique technique assures a more or less rectangular cut through the roots of the lower third molar, which is not the case with the coronal plane and not always with the axial plane.

\section{CLINICAL TESTS}

The proposed oblique scanning technique was clinically tested in patients. For this study, a helical high-resolution MSCT scanner (Philips Medical Systems, Eindhoven, NL) was employed $(175 \mathrm{~mA}, 140 \mathrm{kV})$, with a scanning time of 2 seconds. Patients were recruited based on the surgical need for $3 \mathrm{D}$-imaging prior to scheduled wisdom tooth removal. The data therefore were gained for clinical needs and retrospectively analyzed. The study design fulfills the criteria of paragraphs $4 \mathrm{a}$ and $\mathrm{b}$ according to the guidelines (version 21.5.2010.2010) of the cantonal ethics committee of Zurich and is therefore exempted from institutional review board approval. The study design hence fulfills the guidelines of the Declaration of Helsinki about Ethical Principles for Medical Research Involving Human Subjects. 

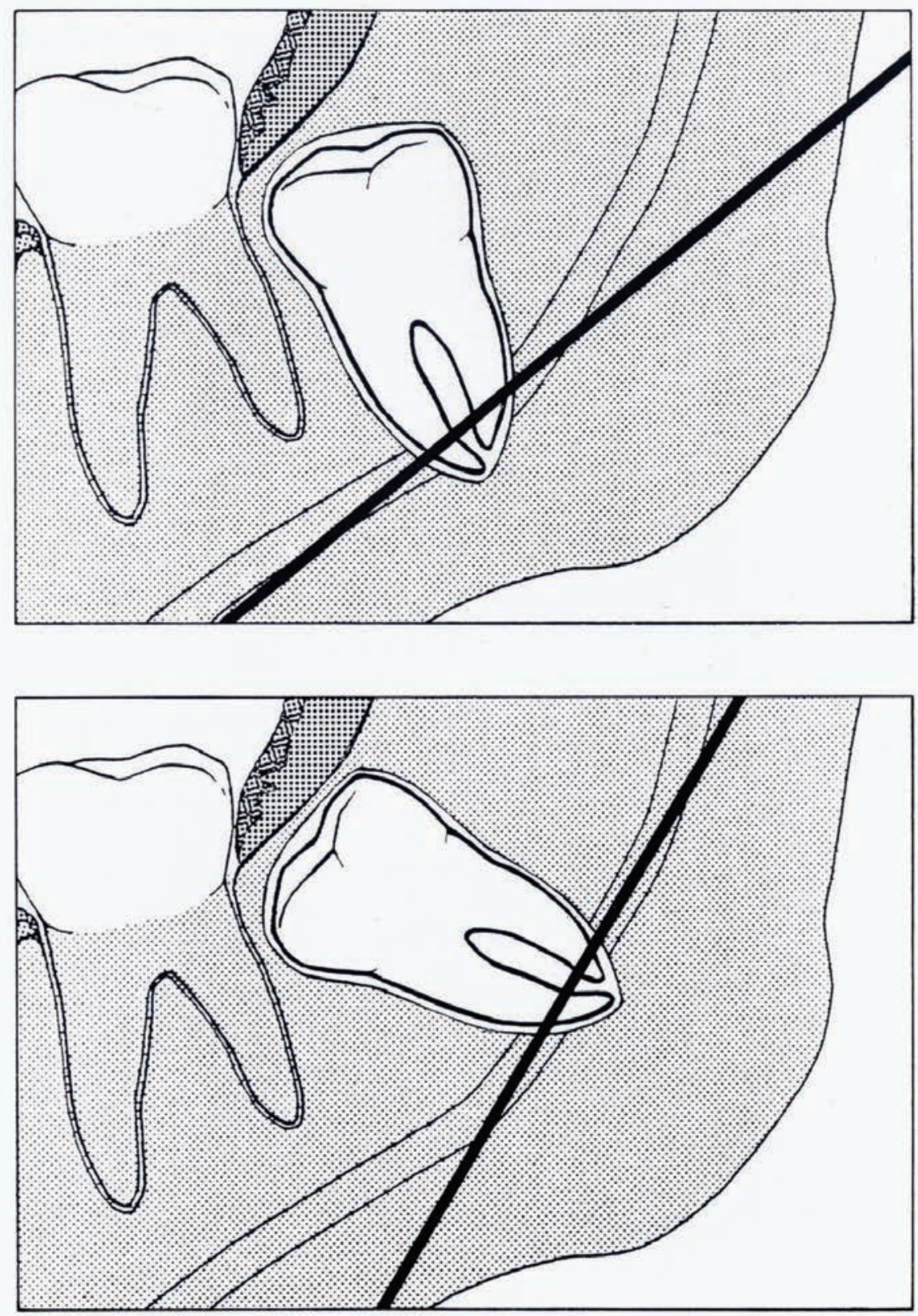

Figure 1. Definition and variations of the oblique plane. The solid lines indicate the oblique planes and their difference in two figures. 

the Gonial Section of the Mandibular Canal and its Related Structures?
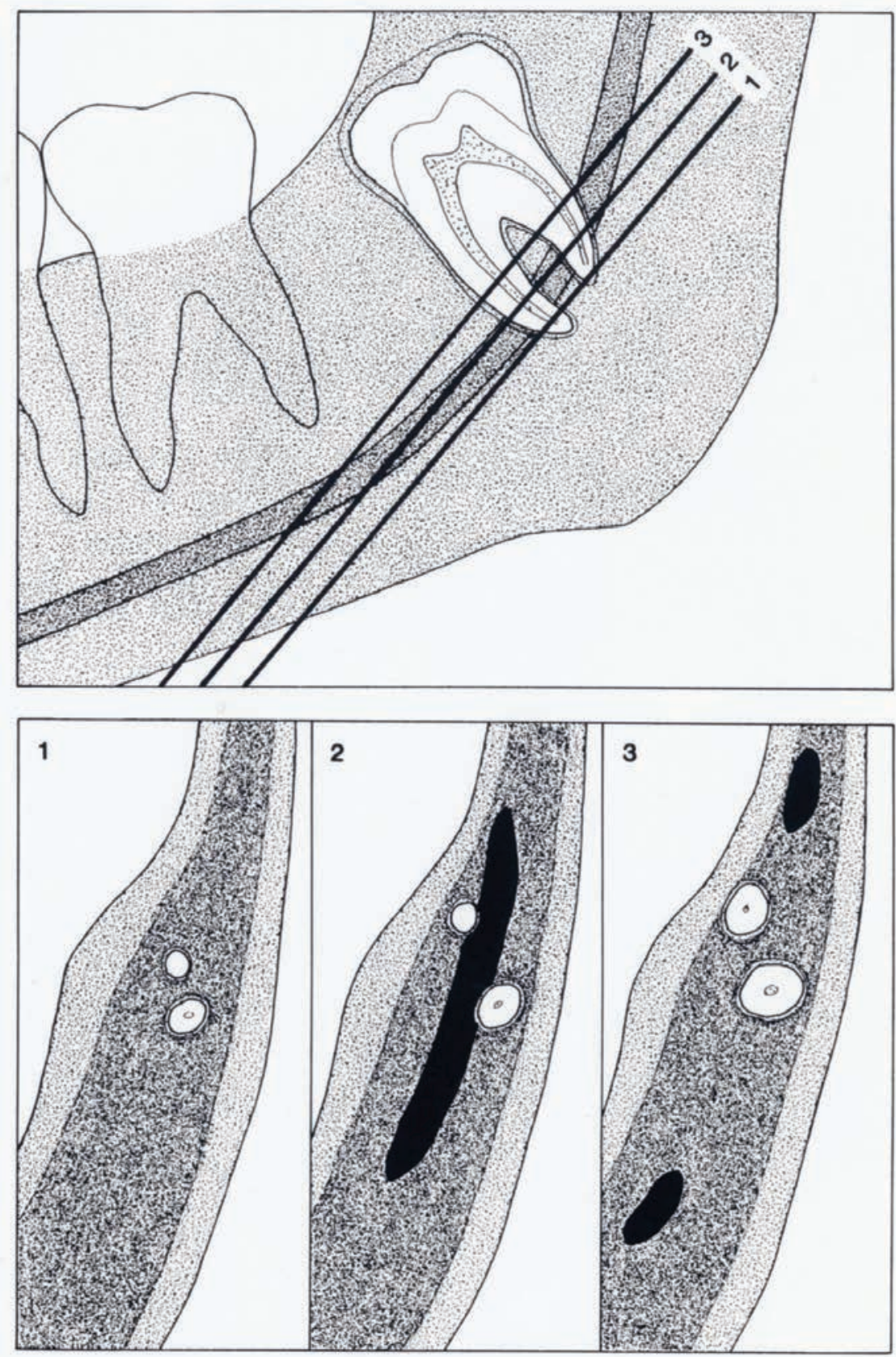

Figure 2a. The oblique plane provides a view of long segments of the nerve canal and a clear overview of the root configuration. 

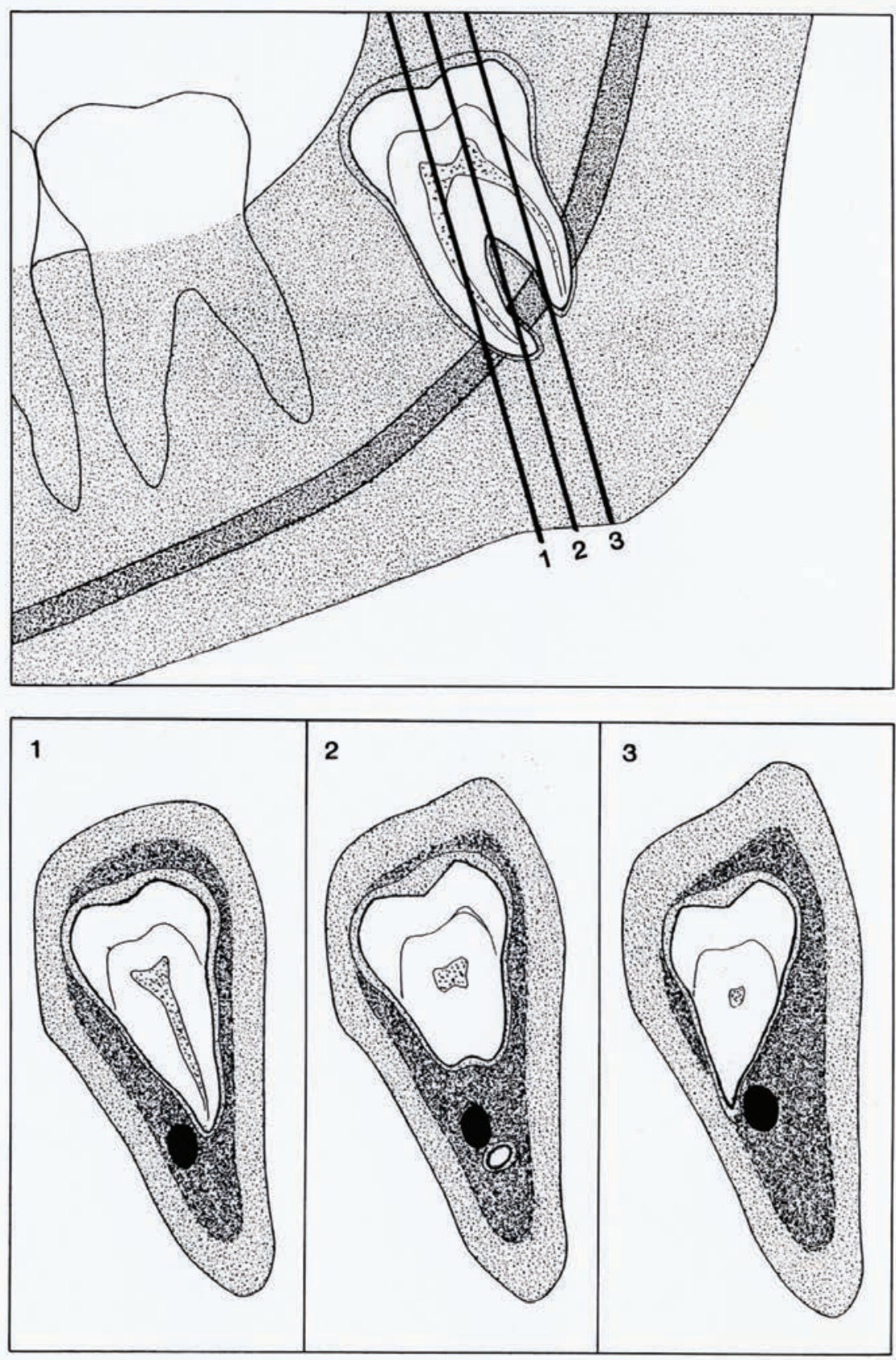

Figure 2b. The conventional coronal plane cannot provide clear visualization of the root configuration. 

the Gonial Section of the Mandibular Canal and its Related Structures?
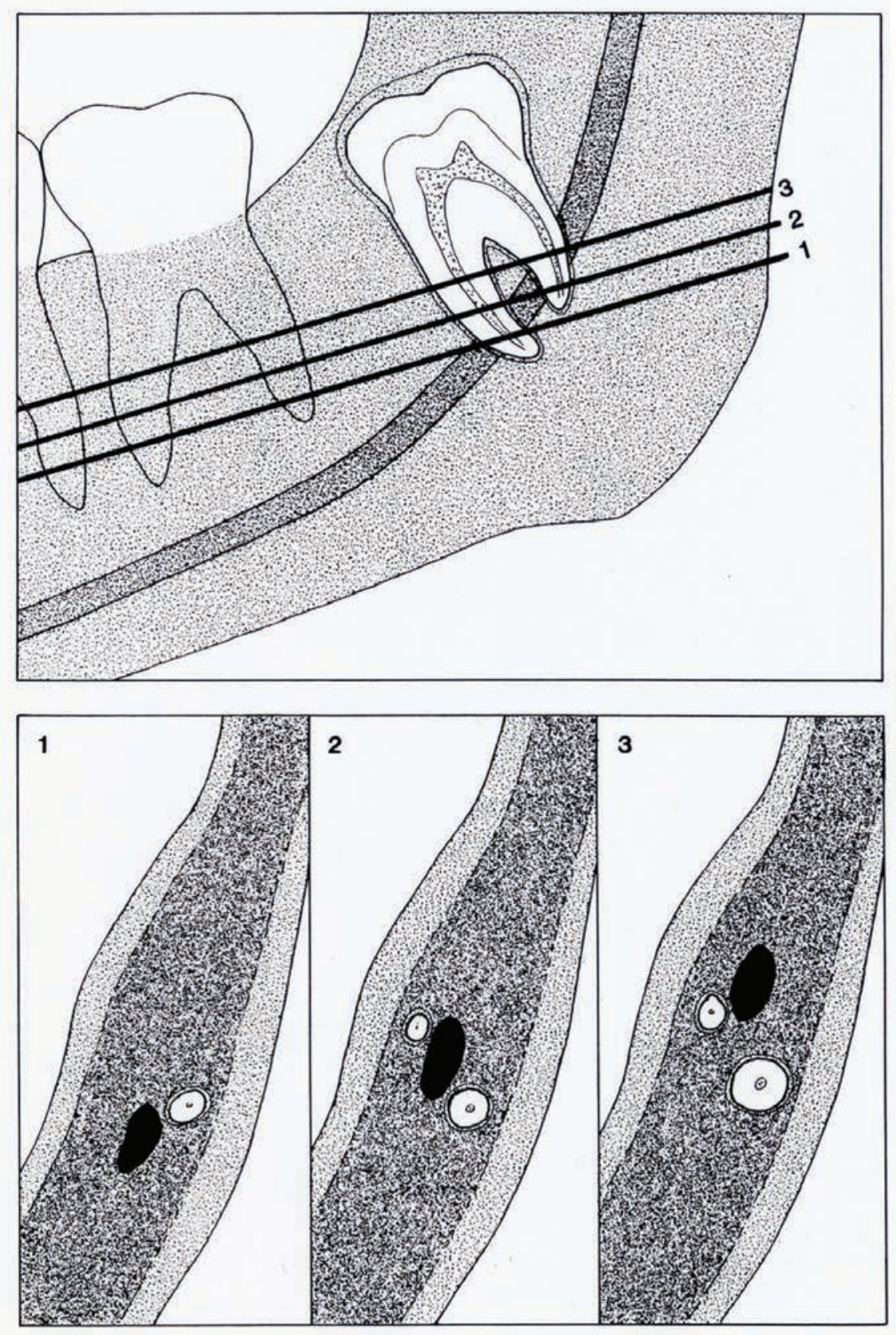

Figure 2c. The conventional axial plane can provide a view of only short segments of the nerve canal. 


\section{RESULTS}

The present study demonstrated that additional information was obtained in four patients by the oblique scanning technique compared to panoramic radiographs and conventional CT-scans.

\section{Patient 1: Removal of wisdom tooth}

A 35-year-old man consulted for removal of an impacted wisdom tooth in the right mandible. The panoramic radiograph showed a projection of the mandibular canal over the roots of the right third molar with the known "high risk" signs of darkening of the root where the canal crossing it and loss of the white cortical outlines indicating a close relationship to the nerve (Figure 3a). Coronal and axial CT scans did not reveal the spatial relationship between the two structures (Figures 3b, 3c). Oblique and tooth-axis scans showed that the alveolar nerve was situated along and between the roots (Figures 3d, 3e).

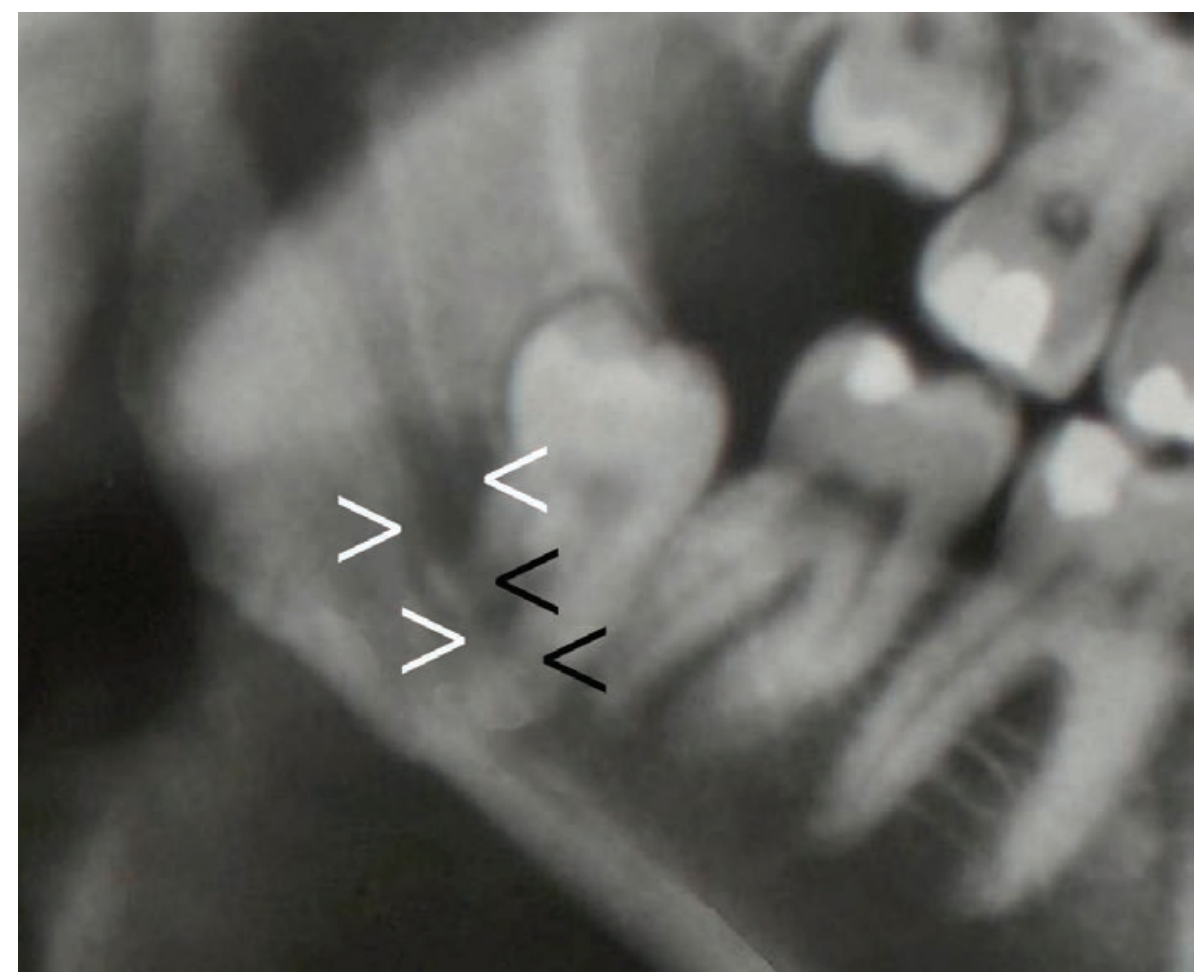

Figure 3a. Magnification of panoramic radiograph suggesting a difficult situation in the lower right wisdom tooth. Darkening of the root where the canal crosses (black arrows) and loss of the white cortical outlines (white arrows) indicate a close relationship to the nerve. 

the Gonial Section of the Mandibular Canal and its Related Structures?

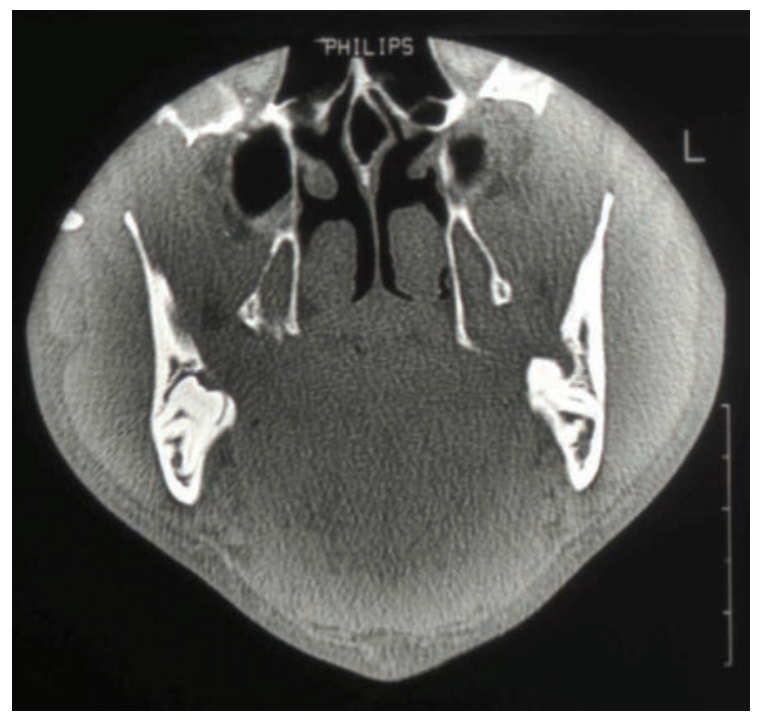

(3b) Coronal view

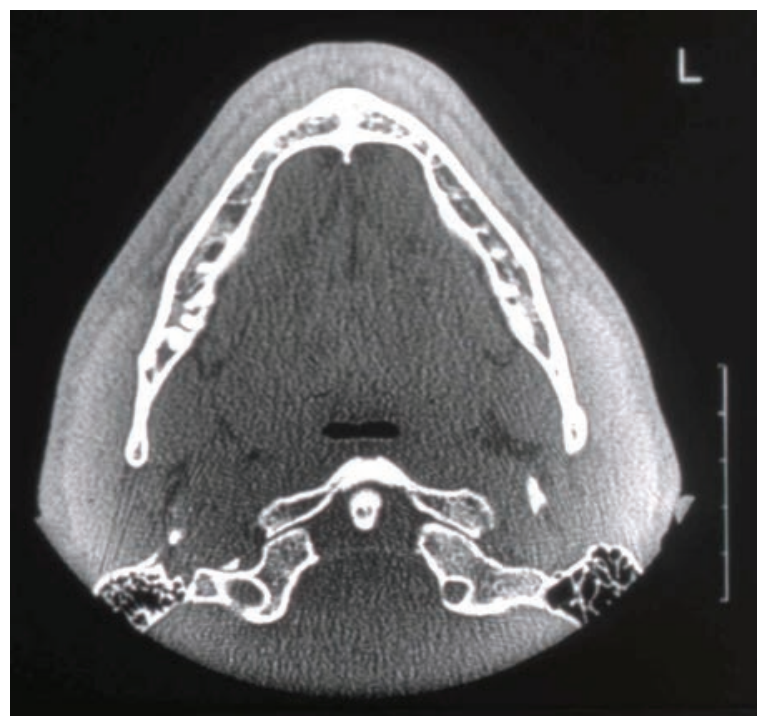

(3c) Axial view

Figures 3b, 3c. The coronal and axial views cannot demonstrate the spatial relationship between the roots and the nerve canal. 


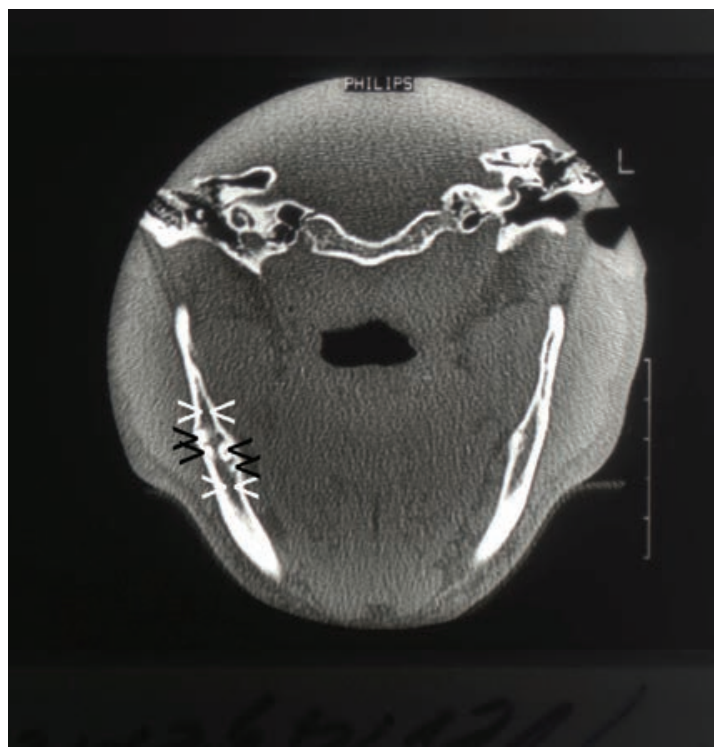

(3d)

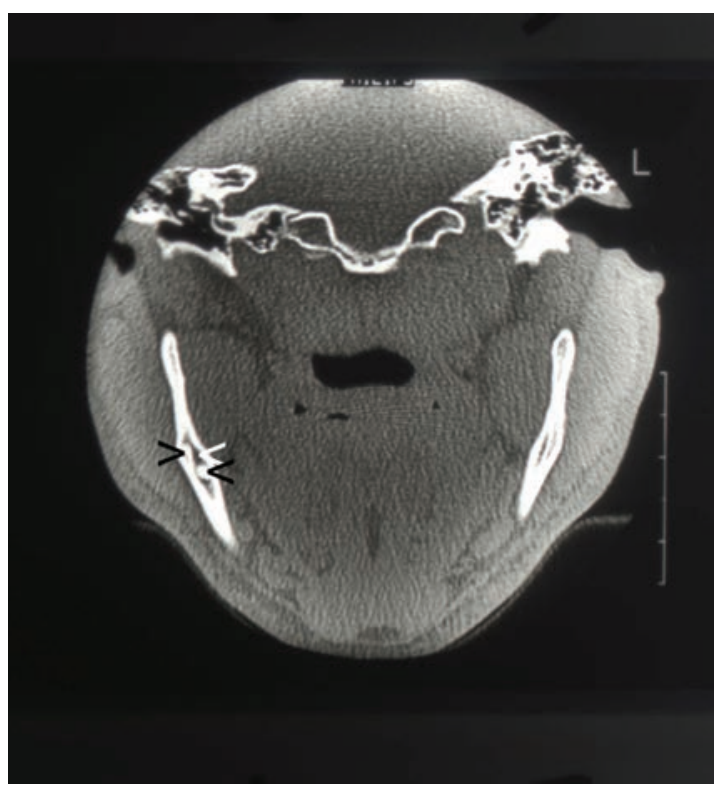

(3e)

Figures 3d, 3e. An oblique view clearly demonstrates the nerve canal (indicated by white arrows) between the roots (indicated by black arrows). 
Patient 2: Diagnosis of undetected pathology causing delayed wound healing A 30-year-old woman suffered from persistent wound-healing impairment following surgical removal of impacted lower wisdom teeth three years ago. The panoramic radiograph showed a bone defect in the third molar region on both sides (Figure 4a). These were also seen on coronal CT scan (Figure 4b), which however did not show the mandibular canal. Further, the coronal CT scan showed a circular structure below the bone defect on the right side, which could be mistaken as the mandibular canal. On the other hand, oblique imaging showed that the mandibular canal was completely surrounded by a tumor (Figure 4c). Surgical biopsy revealed a diagnosis of sarcoidosis.

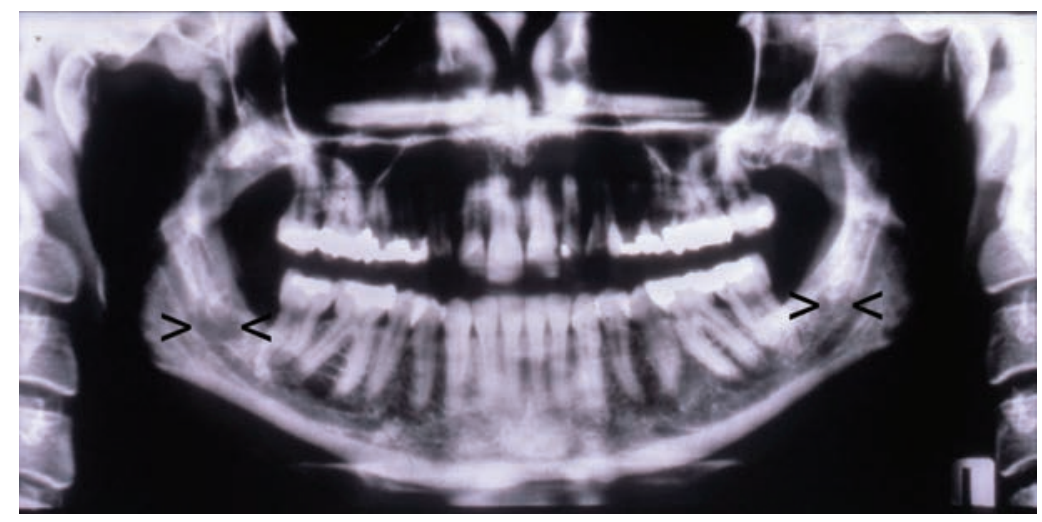

Figure 4a. Panoramic radiograph demonstrates a bone defect in the third molar region on both sides (indicated by black arrows).

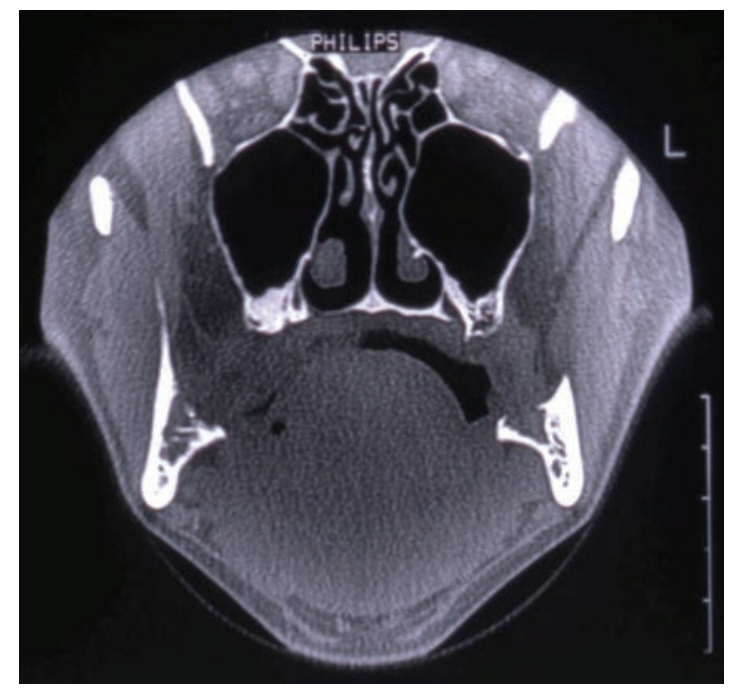

Figure 4b. The coronal plane does not demonstrate the mandibular canal. (Note that the circular structure below the bone defect is not the mandibular canal.) 


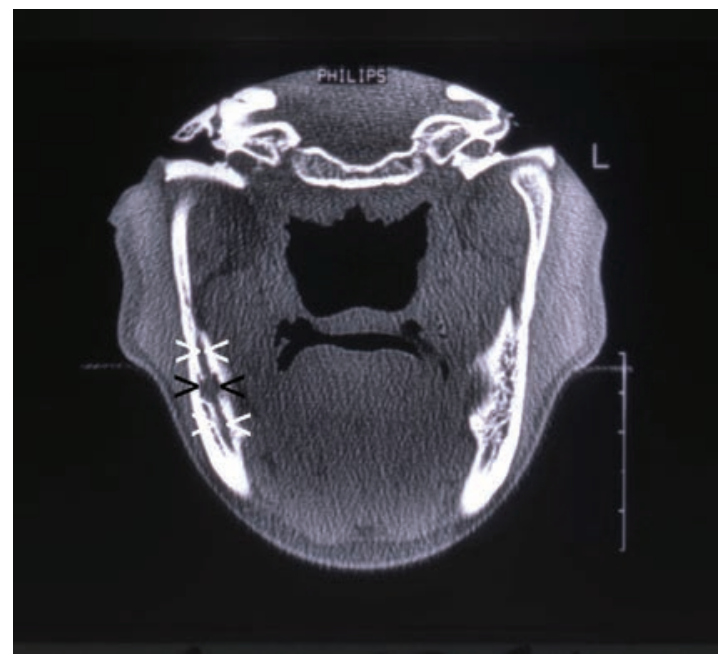

Figure 4c. The oblique scan demonstrates the bone defect (indicated by black arrows) as well as the mandibular canal (indicated by white arrows) running right through the defect.

\section{Patient 3: Detection of foreign body in the mandibular canal}

A 33-year-old woman complained of progressive unilateral paresthesia of the lower lip following surgical removal of an impacted lower wisdom tooth four years ago. Panoramic radiograph showed a radio-dense structure, probably metal, projecting from the mandibular canal (Figure 5a). Because of metal artifact and its limited discrimination from adjacent structures, axial and coronal tomograms did not reveal the relationship between the inferior alveolar nerve and the foreign body (Figures $5 b, 5 c$ ) clear enough. On the other hand, oblique imaging showed a radio-dense object situated in the cranial part of the alveolar canal (Figures 5d, 5e). This was discovered to be a fragment of an amalgam filling, when removed via buccal ostectomy.

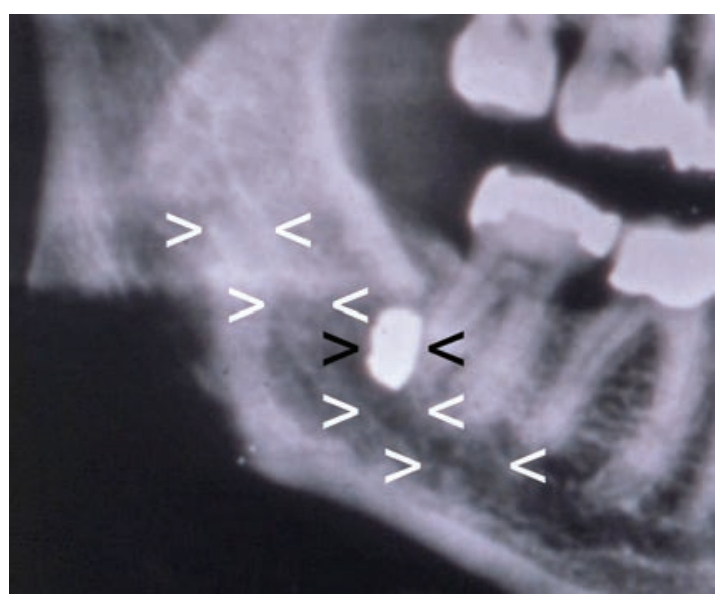

Figure 5a. Magnification of panoramic radiograph suggests a piece of metal (indicated by black arrows) close to the mandibular canal (indicated by white arrows). 

the Gonial Section of the Mandibular Canal and its Related Structures?

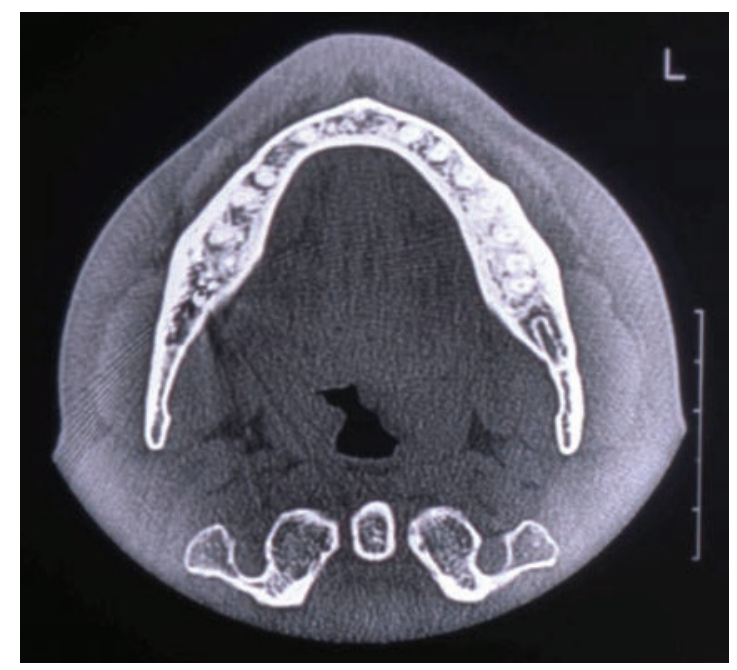

(5b) Axial view

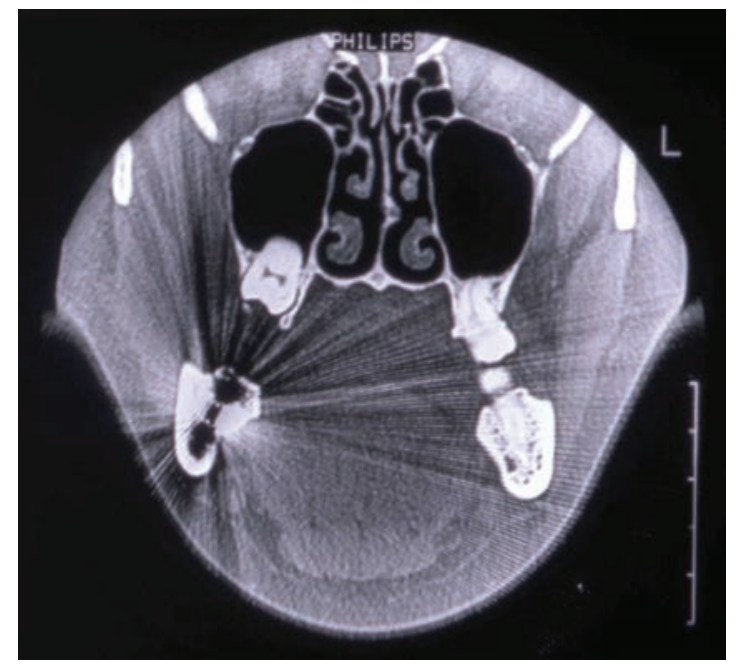

(5c) Coronal view

Figures 5b, 5c. Axial and coronal tomograms cannot provide a clear picture of the relationship due to metal artifact and limited discrimination. 


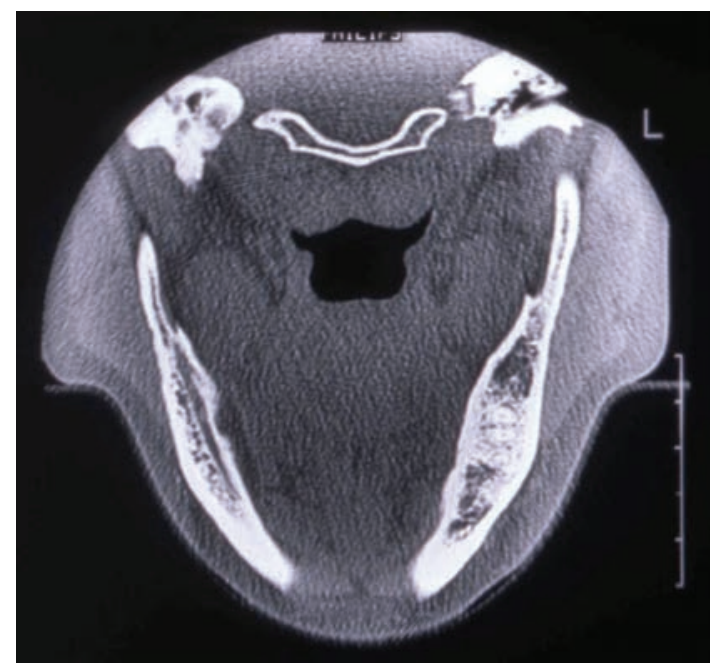

(5d)

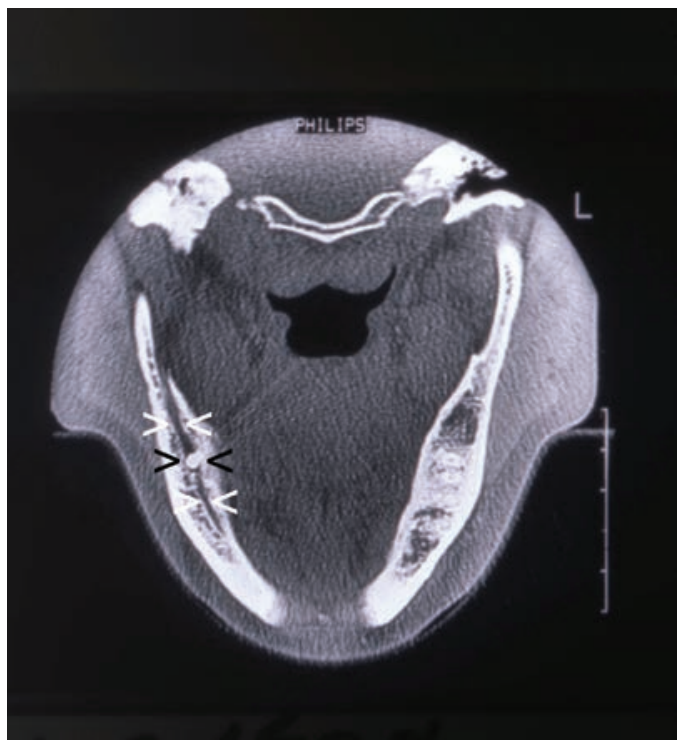

(5e)

Figures 5d, 5e. Oblique tomograms show a radio-dense object (indicated by black arrows) in the cranial part of the mandibular canal (indicated by white arrows). Note that $5 \mathrm{~d}$ shows the inferior part of the mandibular canal which is unharmed by the object. 
Patient 4: Forensic investigation of iatrogenic neurosensitive disturbance of the inferior alveolar nerve

In this case, surgical removal of the left mandibular third molar had caused complete anesthesia of the alveolar inferior nerve. The conventional scans in the axial and coronal planes showed a dental fragment in the socket, but did not allow identification of the path of the mandibular canal and the inferior alveolar nerve at the level of the socket (Figures 6a, 6b). Oblique CT scans showed a dental fragment adjacent to the buccal cortical plate, interruption of the nerve canal, and thickening of the alveolar nerve (Figure 6c).

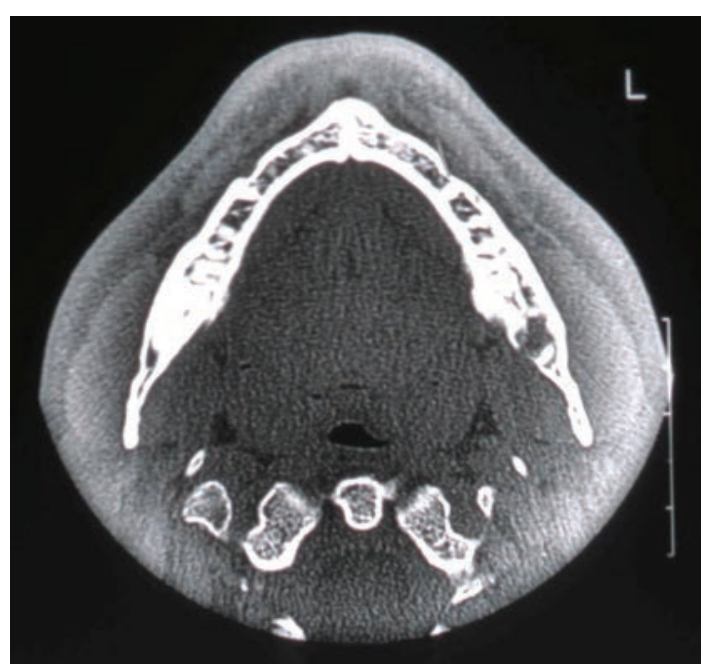

(6a) Axial view

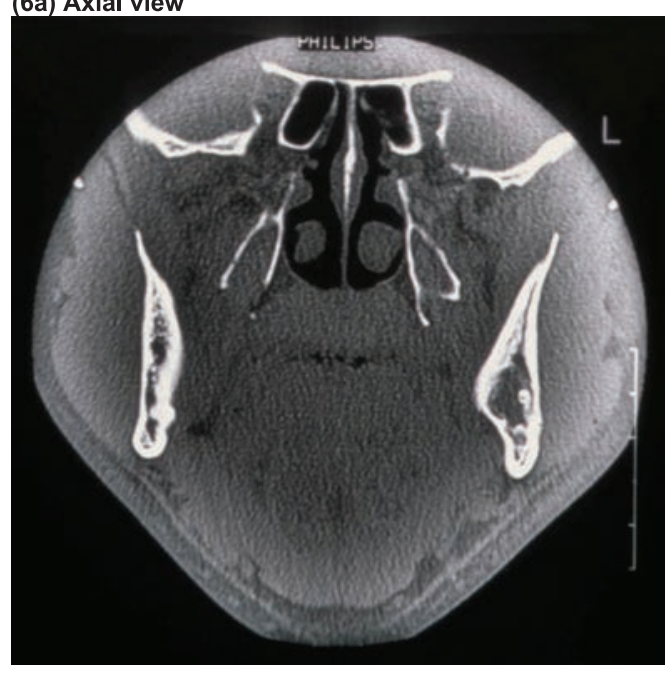

(6b) Coronal view

Figure 6a, 6b. Axial and coronal scans do not allow for identification of the path of the nerve at the socket level. 


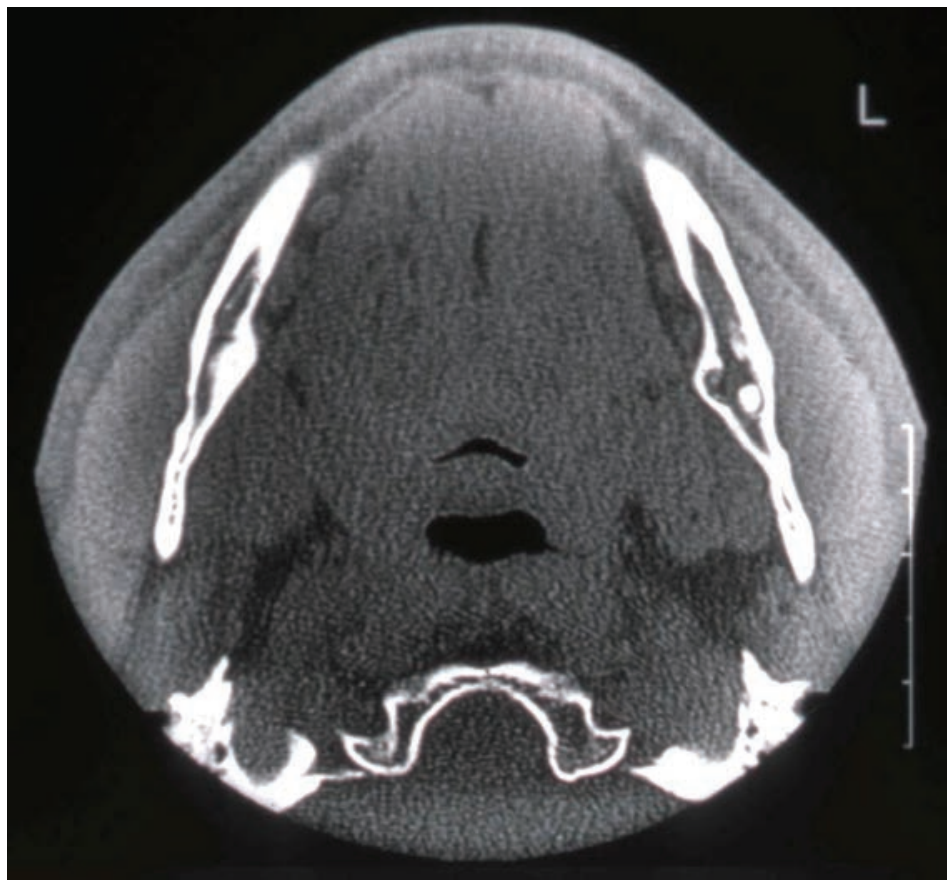

Figure 6c. Oblique tomograms show a radio-dense object adjacent to the buccal plate, interruption of the nerve canal, and thickening of the nerve.

\section{DISCUSSION}

Conventional panoramic radiography provides a two-dimensional image and thus limited information about the mandible and its structures. In wisdom teeth removal, the limitations of these images increase risk [5], as the actual 3D-anatomy of the patient is unknown. Three-dimensional radiography can overcome this limitation and provide additional information, especially concerning the bucco-lingual course of the mandibular nerve $[4,6-8,12,13]$.

The new technique described above provides a high visual quality, which allows an easy and fast assessment of the mandibular canal course through the long cross sections of the nerve canal. The technique also enables avoidance of artifacts and can therefore be a solution in patients with extensive prosthetic reconstructions. The perpendicularity between the cutting plane and the roots gives a good overview of the root configuration. Overall, with the proposed technique, fewer scans will be required and the images are easier to assess (Figure 7).

In our experience and that of trained radiographers, it was possible to achieve an oblique plane acceptable for both sides in most patients. Only patients with extended pathology on one side, such as large cysts deflecting the mandibular nerve canal, show different oblique planes for the right and the left sides. In those cases, most likely the side of interest defines the plane to choose. A small number of patients could not be in 
prone position with neck extended, mainly due to pathologies of the spine. In these patients, supine position mostly solved the problem. It is very seldom that obtaining direct oblique scans was not possible at all.

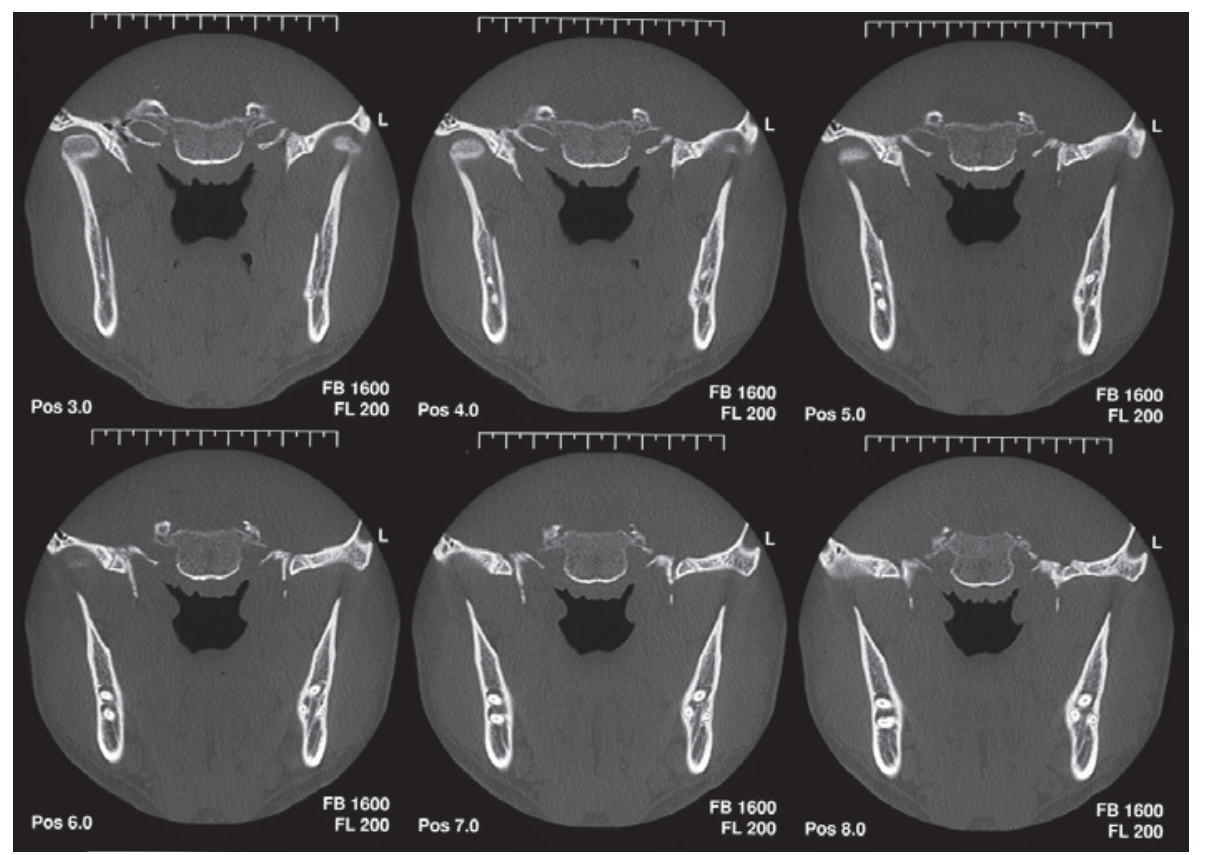

Figure 7. Example for the resulting image quality in only six slices (canal lingual to the roots on the right side, crossing through the root configuration on the left side).

The radiation dose of the described technique is expected to be quite low because of the relatively small height of the volume scanned. To further reduce radiation, the MSCT scanner can also be adjusted to a setting which does not provide soft tissue contrast just like a CBCT[16].

The very small field cannot be achieved by many CBCT scanners because of limitations relative to the patient's position and the factory presettings for the field of view.

The major drawback of this study is the descriptive character by which it can not prove the superiority of the proposed technique. However, we believe the conclusive conceptual idea compensates this issue to a great extent. Certainly, further investigation regarding artifact reduction and diagnostic reliability is obligatory. Especially, the interobserver reliability has to be examined in comparison to other imaging planes routinely utilized for evaluation of the gonial section of the mandible.

Further studies will be necessary concerning the machine-dependent question if MSCT under the special circumstances might even provide a lower radiation dose than a modern CBCT. The dose of the scanogramm of both machines needs to be taken into account as well. Ludlow and Ivanovic did show that radiation dose of CBCT is mostly but not always lower [17]. 


\section{CONCLUSION}

$\mathrm{CBCT}$ is a state of the art solution to 3D-radiography, and modern software tools automatically or semi-automatically mark the mandibular canal. However, in some cases, it is difficult to evaluate the nerve canal and the root configuration. Reorientation of the dataset and displaying the situation in the described oblique plane might be helpful. Particularly, if MSCT instead of CBCT is available, such as in situations with hospitalized patients or in rural regions, the oblique high resolution tomography performed with an MSCT scanner can offer a valuable solution.

Even though this study suggests that the oblique plane scanning is superior to other planes, further studies have to be conducted to determine whether the proposed technique might become the new standard protocol of $3 \mathrm{D}$ evaluation for wisdom teeth.

\section{REFERENCES}

[1] Haug, R.H., Perrott, D.H., Gonzalez, M.L., and Talwar, R.M., The American Association of Oral and Maxillofacial Surgeons Age-Related Third Molar Study. J Oral Maxillofac Surg. 2005, 63(8): 1106-14.

[2] Baqain, Z.H., Karaky, A.A., Sawair, F., Khraisat, A., Duaibis, R., and Rajab, L.D., Frequency estimates and risk factors for postoperative morbidity after third molar removal: a prospective cohort study. J Oral Maxillofac Surg. 2008, 66(11): 2276-83.

[3] Blondeau, F., and Daniel, N.G., Extraction of impacted mandibular third molars: postoperative complications and their risk factors. J Can Dent Assoc. 2007, 73(4): 325.

[4] Susarla, S.M., and Dodson, T.B., Preoperative computed tomography imaging in the management of impacted mandibular third molars. J Oral Maxillofac Surg. 2007, 65(1): 83-8.

[5] Rood, J.P., and Shehab, B.A., The radiological prediction of inferior alveolar nerve injury during third molar surgery. Br J Oral Maxillofac Surg. 1990, 28(1): 20-5.

[6] Nakagawa, Y., Ishii, H., Nomura, Y., Watanabe, N.Y., Hoshiba, D., Kobayashi, K., and Ishibashi, K., Third molar position: reliability of panoramic radiography. J Oral Maxillofac Surg. 2007, 65(7): 1303-8.

[7] Nakamori, K., Fujiwara, K., Miyazaki, A., Tomihara, K., Tsuji, M., Nakai, M., Michifuri, Y., Suzuki, R., Komai, K., Shimanishi, M., and Hiratsuka, H., Clinical assessment of the relationship between the third molar and the inferior alveolar canal using panoramic images and computed tomography. J Oral Maxillofac Surg. 2008, 66(11): 2308-13.

[8] Tantanapornkul, W., Okochi, K., Bhakdinaronk, A., Ohbayashi, N., and Kurabayashi, T., Correlation of darkening of impacted mandibular third molar root on digital panoramic images with cone beam computed tomography findings. Dentomaxillofac Radiol. 2009, 38(1): 11-6.

[9] Ambrose, J., Computerized transverse axial scanning (tomography). 2. Clinical application. $\mathrm{Br} J$ Radiol. 1973, 46(552): 1023-47.

[10] Hounsfield, G.N., Computerized transverse axial scanning (tomography). 1. Description of system. $\mathrm{Br}$ J Radiol. 1973, 46(552): 1016-22.

[11] Engelke, W.G., Diederichs, C.G., and Deckwer, II, Preoperative imaging in a case of molar retention. Int J Oral Maxillofac Surg. 1997, 26(4): 278-9.

[12] Lubbers, H.T., Matthews, F., Damerau, G., Kruse, A.L., Obwegeser, J.A., Gratz, K.W., and Eyrich, G.K., Anatomy of impacted lower third molars evaluated by computerized tomography: is there an indication for 3-dimensional imaging?. Oral Surg Oral Med Oral Pathol Oral Radiol Endod. 2011, 111(5): 547-50.

[13] Eyrich, G., Seifert, B., Matthews, F., Matthiessen, U., Heusser, C.K., Kruse, A.L., Obwegeser, J.A., and Lubbers, H.T., 3-Dimensional Imaging for Lower Third Molars: Is There an Implication for Surgical Removal?. J Oral Maxillofac Surg. 2011.

[14] Jhamb, A., Dolas, R.S., Pandilwar, P.K., and Mohanty, S., Comparative efficacy of spiral computed tomography and orthopantomography in preoperative detection of relation of inferior alveolar neurovascular bundle to the impacted mandibular third molar. J Oral Maxillofac Surg. 2009, 67(1): 58-66. 

the Gonial Section of the Mandibular Canal and its Related Structures?

[15] Tantanapornkul, W., Okouchi, K., Fujiwara, Y., Yamashiro, M., Maruoka, Y., Ohbayashi, N., and Kurabayashi, T., A comparative study of cone-beam computed tomography and conventional panoramic radiography in assessing the topographic relationship between the mandibular canal and impacted third molars. Oral Surg Oral Med Oral Pathol Oral Radiol Endod. 2007, 103(2): 253-9.

[16] Miracle, A.C., and Mukherji, S.K., Conebeam CT of the head and neck, part 1: physical principles. AJNR Am J Neuroradiol. 2009, 30(6): 1088-95.

[17] Ludlow, J.B., and Ivanovic, M., Comparative dosimetry of dental CBCT devices and 64-slice CT for oral and maxillofacial radiology. Oral Surg Oral Med Oral Pathol Oral Radiol Endod. 2008, 106(1): 106-14. 


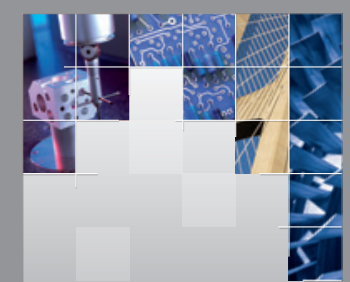

\section{Enfincering}
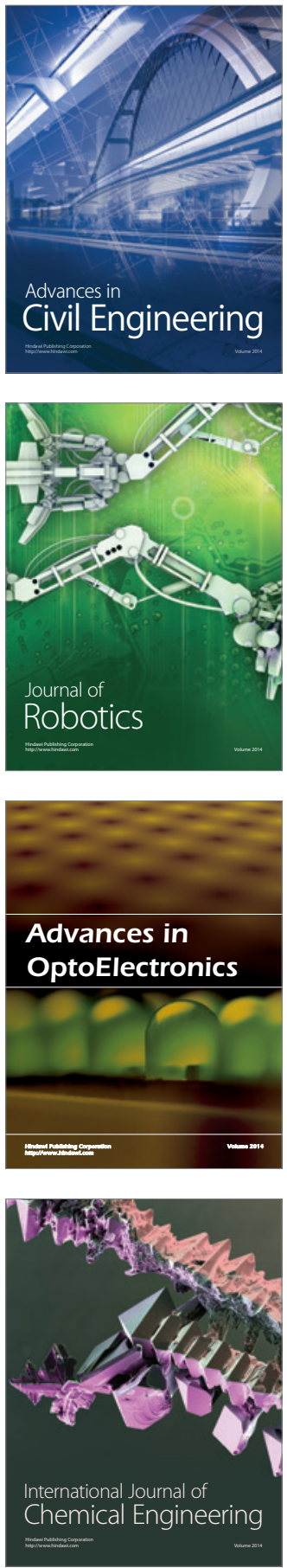

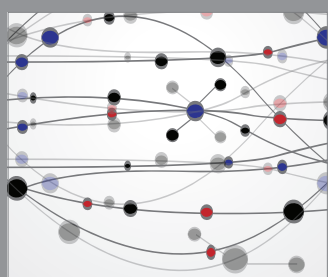

The Scientific World Journal

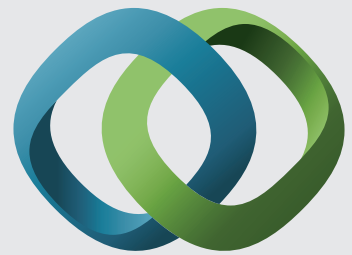

\section{Hindawi}

Submit your manuscripts at

http://www.hindawi.com
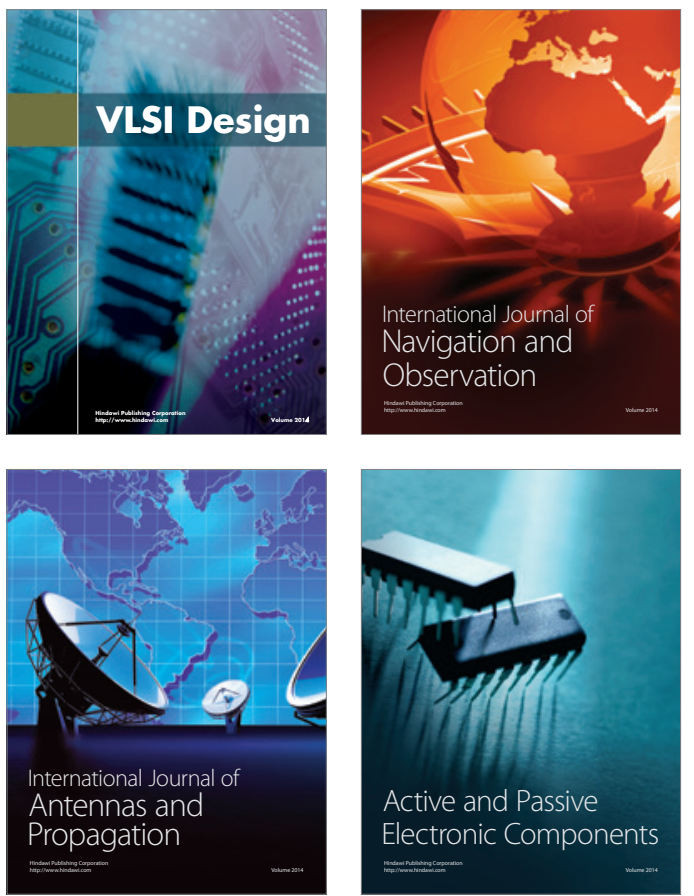
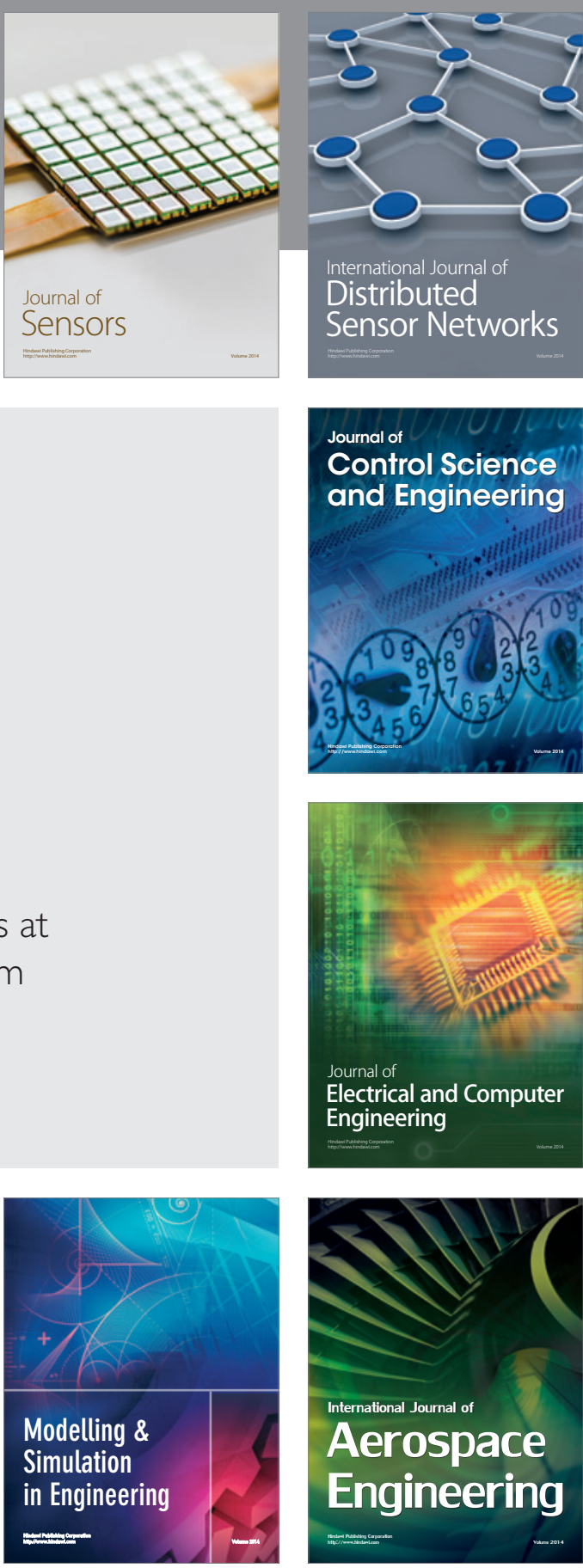

International Journal of

Distributed

Sensor Networks

Journal of

Control Science

and Engineering
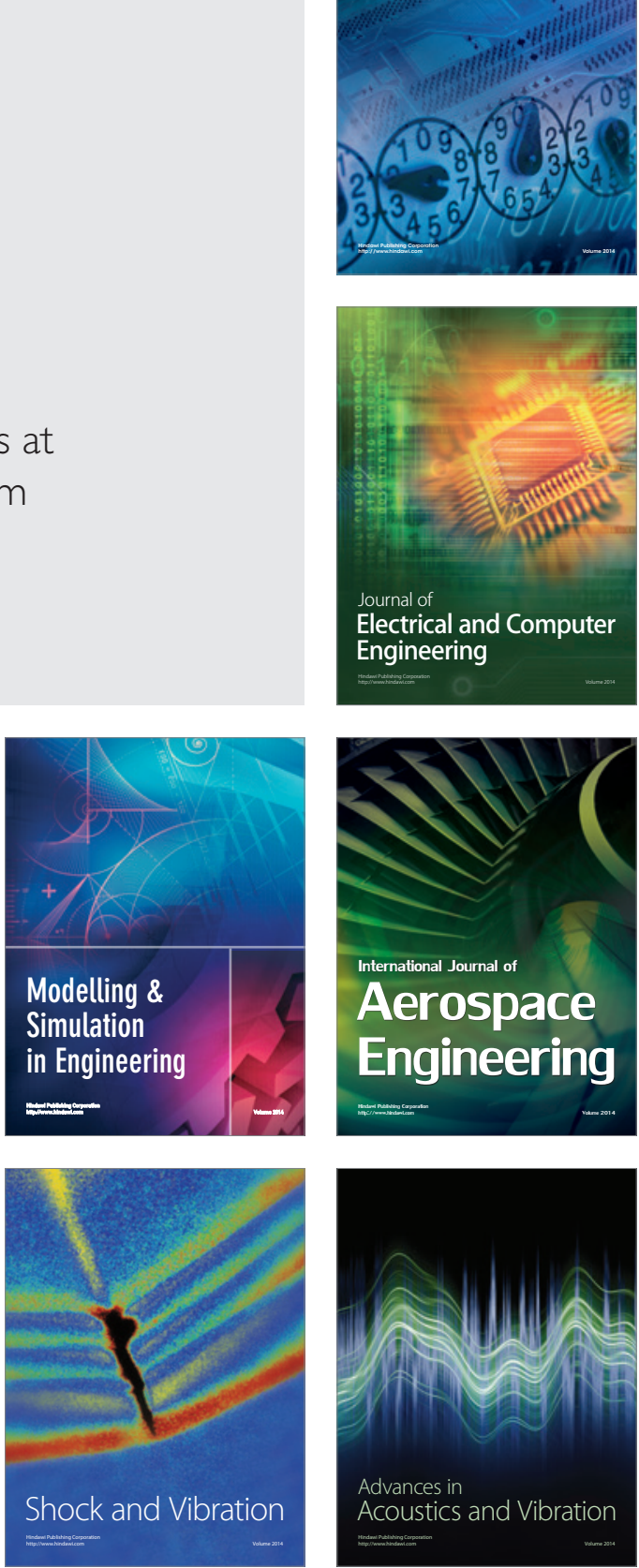